\title{
Cognitive Flexibility Moderates the Efficacy of a Visuospatial Intervention Following Exposure to Analog Trauma
}

\author{
Alla Hemi ${ }^{1}$, M. Roxanne Sopp ${ }^{1}$, Ariel Perel ${ }^{1}$, Emily A. Holmes ${ }^{2,3}$, Einat Levy-Gigi ${ }^{1,4}$ \\ ${ }^{1}$ Faculty of Education, Bar Ilan University, Israel \\ ${ }^{2}$ Department of Psychology, Uppsala University, Uppsala, Sweden \\ ${ }^{3}$ Department of Clinical Neuroscience, Karolinska Institutet, Stockholm, Sweden \\ ${ }^{4}$ The Gonda Multidisciplinary Brain Research Center, Bar Ilan University, Israel
}

We have no conflict of interest to disclose.

The data of this study is available at tinyurl.com/DataTetris

Correspondence concerning this article should be addressed to Einat Levy-Gigi, Faculty of Education and the Multidisciplinary Brain Research Center, Bar Ilan University. Ramat Gan, Israel.

Email: Einat.levy-gigi@biu.ac.il 


\begin{abstract}
Objective: Intrusive memories are the hallmark feature of Post-Traumatic-Stress-Disorder (PTSD). Recent studies have found that a visuospatial intervention after viewing traumatic films reduces intrusive memories in healthy individuals. However, many individuals still exhibit high levels of symptoms following such an intervention, warranting continued investigation into specific characteristics moderating intervention effect. One such candidate is cognitive flexibility, defined as the ability to update behavior in accordance with contextual demands. The present study aimed at examining the interactive effect of cognitive flexibility and a visuospatial intervention on intrusive memories, predicting that higher flexibility would be linked to stronger intervention effects. Method: Sixty participants $\left(M_{\mathrm{age}}=29.07, S D=\right.$ 4.23) completed a performance-based paradigm evaluating cognitive flexibility, watched traumatic films, and were allocated to either an intervention or control group. Intrusions were assessed by means of laboratory and ambulatory assessment, and the Impact of Events Scale - Revised (IES-R). Results: Participants in the intervention group experienced fewer laboratory intrusions than the control group. However, this effect was moderated by cognitive flexibility: Whereas individuals with below-average cognitive flexibility did not benefit from the intervention, it was significantly beneficial for individuals with average and aboveaverage cognitive flexibility. No group differences emerged in the number of ambulatory intrusions or IES-R scores. However, cognitive flexibility was negatively correlated with IES-R scores across both groups. Conclusions: These results highlight the significant role of cognitive flexibility in intrusion development, both in general and as a moderator of a visuospatial interventions. Future studies should investigate effects' generalizability to clinical samples.
\end{abstract}

Keywords: cognitive flexibility, intrusive PTSD symptoms, visuospatial task 


\section{Public Health Significance Statement}

- When treating individuals following traumatic exposure, level of cognitive flexibility may affect choice of treatment.

- For individuals with average and above-average cognitive flexibility, an intervention procedure including a visuospatial game task following an experimental analogue of traumatic exposure reduces intrusive symptoms. 


\section{Cognitive Flexibility Moderates the Efficacy of a Visuospatial Intervention Following \\ Exposure to Analog Trauma}

Post-Traumatic Stress Disorder (PTSD) is a severe mental disorder that may develop after exposure to one or more traumatic events. Individuals suffering from PTSD experience recurrent, involuntary (intrusive) memories of trauma that may trigger reliving and perpetuate perceptions of ongoing threat (Michael et al., 2005). In addition, they may experience hyperarousal, avoidance symptoms, and negative alterations of cognition and mood (American Psychiatric Association, 2013). Longitudinal research indicates that, without treatment, these symptoms are likely to persist across time for some individuals (Zlotnick et al., 1999), thus highlighting the need for effective early interventions (Zohar et al., 2011).

A novel approach aims to prevent the development of intrusive memories by reducing sensory trauma memory in the early aftermath of trauma (Holmes et al., 2009; Holmes et al., 2010). This approach is based on the idea that memories can be updated and rendered less intrusive (James et al., 2015), using a memory reminder cue to make them modifiable. Based on this assumption, Holmes and colleagues have attempted to reduce the occurrence of intrusive memories by reactivating (e.g., using reminder images) and then using a task procedure to compete with mental imagery, that is seeking to modify the sensory memory trace formed during trauma. To this end, after the reminder task and a time gap, they used a visuospatial task based on the game 'Tetris', with specific instructions to engage in mental rotation when placing the blocks (rather than playing Tetris as usual) in order to maximize visuospatial engagement and absorption during the task. Post memory-reactivation exposure to the task procedure aims to selectively interfere with the activated sensory memory trace of trauma and reduce the subsequent emergence of intrusive memories. The efficacy of this task has been tested in several studies, which used traumatic film clips to induce analog PTSD symptoms (Iyadurai et al., 2019), providing evidence of reduced intrusive memories 
following the visuospatial task (Holmes et al., 2009; James et al., 2015). Please note however, that simply playing the computer game Tetris has shown not to be effective in reducing intrusive symptoms (James et al., 2015) - the other parts of the procedure must also be present and conducted in the correct order (James et al., 2016). In addition, neither is "mere distraction" alone sufficient, since studies indicated that some verbal tasks do not have similar effects on intrusive memories as a visuospatial task (Holmes et al., 2010; but see Hagenaars et al., 2017). Moreover, it was shown that the visuospatial intervention procedure can reduce intrusions yet does not affect voluntary explicit memory of trauma (Lau-Zhu et al., 2019; Lau-Zhu et al., 2021). Finally, findings of James et al. (2015) have been independently replicated by Badawi et al. (2020) who received training and feedback on the procedure from the authors of the original study. However, another study with several procedural variations and less training showed null effects (Brennen et al., 2021). This underscores the importance of establishing boundary conditions around an intrusion reduction effect, whether by examining variations in methods' details, or by examining individual differences that may impact the intervention engagement, as in the current study.

Interestingly, even in studies finding an intrusion reduction effect, some individuals do not benefit from the visuospatial intervention (e.g., Kessler et al., 2018). As such, further research is needed to test whether individual characteristics moderate the efficacy of the visuospatial intervention. This approach is in line with the increased efforts to developing personalized PTSD treatments (Katsanis, et al., 2008; Topol \& Lauer, 2003), which aim to address the specific needs and goals of various individual patients. As such, personalized treatment approaches seek to identify critical patient characteristics to which treatment should be tailored to improve outcomes (Delgadillo at al., 2016).

Here we suggest that cognitive flexibility may be such a characteristic. Cognitive flexibility refers to the ability to adapt behavior to changing contextual demands (Cañas et al., 
2006; Dennis \& Vander Wal, 2010; Geurts et al., 2009; Ionescu, 2012; Keith et al., 2015). It enables individuals to shift between discrepant tasks and emotional demands and adapt to different challenges (Koesten et al., 2009; Leung \& Zakzanis, 2014). A specific component of cognitive flexibility is the ability to update outcome expectations based on changes of contextual features of the environment. This ability is assumed to be critical for recovery from trauma since re-evaluating and specifying expectations concerning danger that were formed during trauma constitutes one of the major challenges during posttraumatic adjustment. That is, during trauma, individuals learn to associate benign environmental stimuli with the imminent danger of trauma (Kube et al., 2020). When faced with harmless yet similar - stimuli in their post trauma environment, it is critical for their successful recovery to learn that these stimuli have changed their meaning and are now associated with safety.

In line with this assumption, extensive work from Levy-Gigi and colleagues shows that the ability to learn that stimuli previous predictive of negative outcomes are associated with positive outcomes (i.e., negative-to-positive updating) is linked to and modulates PTSD symptoms (Haim-Nachum \& Levy-Gigi, 2021; Levy-Gigi et al., 2015; Authors, submitted; Zabag et al., 2020). Moreover, effects are specifically found for context updating, which converges with the assumption that updating of expectations associated with contextual features of the environment is particularly critical for recovery (Garfinkel et al., 2014). Deficits in updating of contextual features has further been proposed to tie in directly with the occurrence of intrusive memories. That is, the failure to use contextual information (indicating a harmless environment) for updating is considered critical for PTSD symptom development, since such failure is assumed to decreases the precision of incoming sensory input, giving rise to vivid re-experiencing of trauma in the form of intrusive memories (Kube et al., 2020). 
Beyond its role in successful recovery from trauma, flexible updating may also affect the efficacy of visuospatial interventions. On the one hand, flexible updating encompasses the ability to disengage from previous expectations based on changes in environmental sensory input. Correspondingly, it is necessary to disengage from reactivated traumatic content and focus attention on the visuospatial task procedure for the intervention to be successful. On the other hand, the successful modification of memory traces during reactivation may tap into similar updating processes that are involved in updating outcome expectations. Despite these potential links, to date no study has examined whether individual differences in updating flexibility affect the efficacy of the visuospatial intervention. Based on the considerations laid out above, average and above-average updating flexibility should be associated with a significant impact of the visuospatial intervention on intrusive memories. By contrast, low updating flexibility should hinder the intervention from being effective, resulting in a lack of significant effects on intrusive memories. Irrespective of intervention effects and based on previous frameworks (Kube et al., 2020), a significant negative association should emerge between updating flexibility and intrusive memories.

To test these assumptions, the present study examined the interactive effects of cognitive flexibility and a visuospatial intervention procedure (i.e., playing Tetris after a memory reminder using metal rotation) on levels of intrusive memories after exposure to a traumatic film. Pre-film cognitive flexibility was assessed using a performance-based updating task, with high flexibility being defined as a high accuracy in negative-to-positive context updating (see Levy-Gigi et al., 2015). We hypothesized that individuals in the intervention condition would experience fewer intrusive memories compared to individuals in the control condition. Moreover, we predicted that these effects would only be evident for individuals with average or above-average cognitive flexibility. Finally, we hypothesized that 
- across both groups - higher levels of cognitive flexibility would be associated with lower levels of intrusive symptoms.

\section{Method}

\section{Participants}

A total of 60 young adults ( 40 females, 20 males; age range $=18-35$ years, $M=$ 29.07, $\mathrm{SD}=4.23$ ) were recruited via advertisements at the university. All participants had normal or corrected to normal vision, with no current or past psychiatric disorders, history of concussion or other clinically significant head injury or neurological disorders (such as epilepsy, multiple sclerosis, stroke, or encephalitis). The study was approved by the ethics committee of the authors' institution (Approval \#115). Participants gave their written and informed consent before beginning the study and were reminded that they could leave the experiment at any point with no penalty. At the end of the experiment participants were debriefed.

\section{Measures}

The Cue-Context Reversal Task. We used a well-validated performance-based paradigm with a unique partial reversal design (e.g., Haim-Nachum \& Levy-Gigi, 2019, 2021; LevyGigi et al., 2014; Levy-Gigi et al., 2015; Levy-Gigi et al., 2019; Zabag et al., 2020). In this paradigm, participants view a series of boxes on a computer screen. On each box there is a picture of a target cue (e.g., a hat) presented against a background context (e.g., orange color). When opened, each box is associated with a positive or a negative outcome. The paradigm has two phases: acquisition and retention-reversal phases. In the first phase, participants see a set of boxes, each composed of a unique cue and a unique context. Through trial and error, they learn which box predicts a positive- and which predicts a negativeoutcome (i.e., a box with a hat on an orange background has gold inside while a box with a 
car on a yellow background has a bomb inside). In order to complete the acquisition phase and move on to the next phase, participants had to complete a minimum of 40 trials and reach a criterion of 6 continuous correct responses. In the second phase, in addition to the original boxes (retention trials), participants see boxes that share either the same cue with the original box (e.g., a hat against a grey background), or the same context with the original box (e.g., a phone against an orange background) but have the opposite outcome. Therefore, in order to successfully learn these new associations, participants need to reverse the association rule of either the original cue or the original context. The phase contains 40 retention and 80 reversal trails. Based on previous research on flexibility in trauma and PTSD, (Haim-Nachum \& Levy-Gigi, 2019, 2021; Levy-Gigi et al., 2015; Levy-Gigi \& Richter-Levin, 2014; Zabag et al., 2020), we defined cognitive flexibility as the ability to reverse context-related information from negative to positive (negative-to-positive context updating).

Trauma film. The 12-min trauma film consisted of 11 different scenes involving actual or threatened death, as well as serious injury. Scenes contained different types of contexts; examples include a young girl hit by a car with blood dripping out of her ear, a man drowning in the sea, and a van hitting a teenage boy who was using his mobile phone while crossing the road. This film footage has been used in previous studies to evoke intrusive memories (Holmes et al., 2009; Holmes et al., 2010; James et al., 2015).

Memory-reactivation task. The memory-reactivation task consisted of two parts: (a) presentation of film stills and (b) a 10 min break with a standardized filler task to allow time for memory updating processes to be initiated (Ågren et al., 2012; James et al., 2015). Film stills included 11 static visual images, one from each of the scenes in the film that were presented for a duration of $2 \mathrm{sec}$. Images were taken from the moment just prior to the worst part of a given scene, which typically yield intrusive memories (James et al., 2015; Michael \& Ehlers, 2007). For examples a young girl sitting at the side of the road (just before she was 
hit by a car), a man striding through the sea (just before he drowns), and the face of a teenage boy smiling at the camera (just before he was hit by a van). The images were presented in the same order as the scenes had been shown in the film. During the following 10-minute break, participants conducted a standardized filler music task, in which excerpts of classical music were rated for pleasantness.

Visuospatial task ('Tetris'). The PC game 'Tetris' is comprised of seven differently shaped and brightly colored geometric blocks (e.g., a blue rectangle, an orange T shape), that fall from top to bottom of the playing screen in a random sequence one at a time. Using the keyboard arrow keys, players can move the blocks left or right, rotate them $90^{\circ}$, or accelerate them down as they fall to the bottom of the playing screen. The purpose is to create complete horizontal lines across the playing area using the blocks. Once a full horizontal line is created, it disappears, and the participant receives points. During the current experiment, participants were asked to focus on the three next blocks due to fall (these blocks were displayed in a preview to the right of the playing screen). To encourage mental rotation, participants were asked to work out in their "mind's eye" where best to place these blocks in order to create the horizontal lines and receive points. The game was played in a sound-off mode and the game score for each participant was recorded $\left(\mathrm{M}_{\text {score }}=22010.07, \mathrm{SD}=\right.$ 23243.98, Range 1005 - 88901). The version of Tetris used was 1.2.1 (Blue Planet Software, 2007) with Marathon mode on. Immediately after the game, participants were asked to rank how difficult they perceived the game from 1 - "Not difficult at all/Easy" to 10 - "Extremely difficult/Hard" (M $\mathrm{M}_{\text {perceived difficulty }}=3.10, \mathrm{SD}=2.94$, Range 1 - 9).

\section{Outcome variables}

Laboratory intrusions. Stimuli for the Intrusion-provocation task (IPT) consisted of 11 blurred static visual images, one from each traumatic scene, created using GIMP (Version 2.1.1) software (Gaussian Blur set at 2.0). Images were presented, for $2 \mathrm{sec}$ each, in a mixed 
random order on a white background. Following the presentation participants were instructed to press a button for any intrusive memory the film triggered for a 2-minute duration. The total frequency of pressing the button comprised the IPT intrusion score (Holmes et al., 2009; James et al., 2015; Lau-Zhu et al., 2019, 2021).

Ambulatory intrusions. Participants were given a paper diary to record in writing any intrusive memory of the film content they had in their daily life (outside the lab) for the first $24 \mathrm{hr}$ (Day 0) and again for Days 1 to 7 (Holmes et al., 2004; Holmes et al., 2009; James et al., 2015). Participants were explained (and received written instructions in the diary) that intrusive memories were defined as scenes of the film that appeared spontaneously in their mind. They were asked not to include memories that they deliberately recalled. Participants were also instructed to include only intrusive memories that included image-based content (e.g., "in the form of pictures in your mind's eye"). They were asked to describe the content of each of their intrusions in the diary (e.g., a silver car crushing couple against a wall) so that the experimenter could later confirm whether or not the intrusive memories were related to the film. Each day of the diary was labeled and split into three sections (morning, afternoon, and evening), and participants were asked to mark the specific section when they experienced an intrusive memory (or to indicate that they had not), and then to write the content of the intrusion. They were asked to record all intrusions immediately. If participants had experienced no intrusions during any time period, they were asked to enter zero in the diary. At the end of the experiment, participants were asked to rate how accurately they have completed their diary.

Impact of Event Scale-Revised (IES-R). The Intrusion subscale (Weiss \& Marmar, 1997) comprised 8 items that were in reference to the film (e.g., "Pictures about the film popped into my mind"). Participants were asked to indicate how distressing each item was "during 
the past 7 days with respect to the film you watched last week." Items were rated on a 5-point Likert scale from 0 (not at all) to 4 (extremely).

Recognition memory tests. The verbal recognition memory test consisted of 32 true/false written statements relating to the 11 scenes in the trauma film (e.g., "Scene 1: The little girl has blood coming from one of her nostrils" and "Scene 7: A man swims out to retrieve an inflatable lilo [beach air mattress]". The visual recognition memory test comprised 22 static visual images; half taken from throughout the film ( 1 per scene, different images from those used in the IPT), and half were previously unviewed images. Images were presented individually for $5 \mathrm{~s}$ each. For both visual and verbal recognition memory tests, participants indicated yes or no (on paper) as to whether or not the image (visual recognition memory test) or written statement (verbal recognition memory test) was recognized by them as being from the film watched 8 days earlier.

PTSD Checklist for DSM-5 (PCL-5). The PCL-5 is a civilian version that comprises a 20item checklist of PTSD symptoms closely based on the DSM-V criteria. Each item is scored on a 5-point scale ranging from 0 to 4 , with a total range from 0 to 80 , with higher scores reflecting greater levels of PTSD symptoms (Blevins et al., 2015).

\section{Control variables}

Beck Depression Inventory (BDI-II). A 21-item self-report questionnaire designed to measure depressive symptoms (Beck, Steer, \& Brown, 1996). Each item is scored on a 4point scale ranging from 0 to 3 , with total scores ranging from 0 to 63 ; higher scores indicating greater levels of depression. The BDI-II has high internal consistency in clinical outpatients $(\alpha=.92)$ and in student samples $(\alpha=.93$; Beck, Steer, Ball, \& Ranieri, 1996). State-Trait Anxiety Inventory (STAI-T). The STAI-T is a 20-item self-report measure designed to measure state anxiety and trait anxiety (Spielberger et al., 1983). Each item is 
rated on a 4-point scale, with scores ranging from 20 to 80 ; higher scores represent greater levels of anxiety.

\section{Procedure}

After signing the consent form, all participants completed the cue-context reversal task. In the first session (Day 0) participants viewed the film, and in the second session (Day 1), they were assigned to one of the two experimental group. The first two sessions were conducted $24 \mathrm{hr}$ apart and the third session occurred a further 7 days later (Day 7). Participants filled a handwritten paper diary at home to record the daily frequency of intrusive memories throughout the week following watching the trauma films.

Prior to the second session, participants were randomly assigned to one of two groups (visuospatial intervention group: $\mathrm{n}=30$; no-task control group: $\mathrm{n}=30$ ). All participants were given the memory-reactivation task, before which they were told, "I am about to show you a selection of pictures from the film you watched yesterday. I would like you to sit still and pay close attention. Do not look away, and really immerse yourself in viewing the pictures". Following the memory reactivation task there was a time gap of 10 min during which participants were asked to wait before the next task was presented. (Ågren et al., 2012). Following the memory-reactivation task and the time gap, participants in the intervention group were given instructions for playing the Tetris game. They were reminded to focus on the three blocks due to fall after the one that they were currently manipulating. To encourage mental rotation, participants were asked to work out in their "mind's eye" where best to place these blocks to create the horizontal lines and be awarded points. Following these instructions, the participants played the Tetris game for $12 \mathrm{~min}$. During this 12-minute period, participants in the control group were asked to sit quietly for $12 \mathrm{~min}$ and received the following instructions "There will now be a short break. Please stay seated and do not talk to the experimenter during this period. You can think about anything, with no restrictions." All 
participants were then asked to continue keeping the diary daily for another seven days. Seven days later (on Day 7), all participants returned to the laboratory for the third session with their completed paper diary (ambulatory intrusions measure). They were asked to rate how accurately they had completed their diary (diary compliance) from 1 (not at all accurately) to 10 (extremely accurately) and completed the laboratory intrusions measure (IPT) and the verbal and visual recognition memory tests for trauma film content and the IES-R Intrusion subscale.

\subsection{Statistical analysis}

Prior to conducting statistical analyses, we inspected the distribution of the intrusion measures for potential outliers. Outliers were defined as individuals whose scores exceeded +/- 3 SD from the group mean. Using this criterion, we identified one outlier for laboratory intrusions. However, since the exclusion of this outlier did not change the results, the entire data set was analyzed.

We used t-tests for between-groups comparisons of baseline characteristics (PCL-5 score, depression, anxiety, age), intrusive memory frequency across the first $24 \mathrm{hr}$ (prior to the visuospatial intervention), intrusive memory frequency across Days 1 to 7 (postintervention), laboratory intrusions (IPT), IES-R intrusion subscale, visual and verbal recognition memory scores, attention to the film, film distress, and diary compliance. Gender was compared between groups using a chi-square test. To assess mood deterioration resulting from viewing the trauma film, we conducted a two-way repeated measures analysis of variance (ANOVA) with main factors of time (pre- vs. post- film) and group (visuospatial intervention vs. control).

For each intrusion measure that revealed significant between-group differences, we next conducted moderation analyses to assess the impact of cognitive flexibility on intervention effects. To this end, we used the PROCCESS macro for SPSS (Hayes, 2017) 
using 5,000 bootstrap resampling for calculation of confidence intervals. Group (visuospatial intervention vs. control) was introduced as independent variable, cognitive flexibility served as moderator, and intrusive memories as dependent variable. To account for group differences in verbal recognition memory, we entered the respective variable as covariate. All predictors were z-standardized for better interpretation.

Finally, we investigated associations between cognitive flexibility and intrusive memories by calculating partial correlations between cognitive flexibility, ambulatory intrusions, laboratory intrusions (Day 0-7), and IES-R scores while controlling for group effects. Two-tailed tests and an alpha level of .05 were used for all statistical analyses. The data is available in an online repository.

\section{Results}

\section{Baseline group comparisons and manipulation checks}

No baseline differences were found between the two groups in terms of age, $t(57)=$ $1.32, p=.191, d=0.346$, depressive symptoms, $t(58)=1.45, p=.152, d=0.375$, general PTSD symptoms, $t(58)=0.74, p=.460, d=0.193$, trait anxiety, $t(58)=1.05, p=.300, d=$ 0.27 , or gender, $\chi^{2}=0.00, p=1.00$ (Table 1$)$.

Mood was equivalent between groups, $F(6,53)=1.24, p=.31, \eta_{p}^{2}=0.123$, and deteriorated from pre- to post-film, $\mathrm{F}(6,53)=10.41, \mathrm{p}<.001, \eta_{p^{2}}=0.541$, with this deterioration being comparable between groups, $\mathrm{F}(6,53)=1.24, \mathrm{p}=.31, \eta_{p}{ }^{2}=0.124$ (Table 2). Moreover, both groups reported similar levels of film attention, $t(58)=0.10, p=.917, d=$ 0.032 , film distress, $t(58)=1.80, p=.077, d=0.465$, and diary compliance, $t(58)=0.32 p=$ $.754, d=0.082($ Table 3$)$.

\section{Analog symptoms of post- traumatic stress}


As reported in Table 3, for the first day prior to the visuospatial intervention, the two groups reported a similar number of ambulatory intrusive memories, $t(58)=0.98, p=.331, d$ $=0.253$.

Following the intervention, participants in the intervention group reported fewer laboratory intrusions than did those in the control group, $t(58)=3.40, p=.001, d=0.878$. There were no significant differences between groups in the number of ambulatory intrusions (days $1-7), t(58)=1.02, p=.312, d=0.264$, or IES-R scores, $t(58)=1.53, p=.131, d=$ 0.396, see Table 3

\section{Recognition memory test}

A recognition memory test given after one week, revealed no differences between the groups in visual memory of the trauma film, $t(58)=1.19, p=.238, d=0.308$. However, verbal recognition memory was significantly lower in the intervention group, $t(58)=3.89, p$ $<.001, d=1.003$. Since prior studies by Holmes and colleagues (2009) found visual and verbal recognition memories comparable after the visuospatial intervention, we added verbal recognition memory as a control variable in further analyses.

\section{Cognitive flexibility as a moderator of group difference in intrusive memories}

To test the hypothesis that cognitive flexibility moderates the impact of the intervention (visuospatial intervention vs. control) on intrusive memories, Hayes's (2017) PROCCESS macro was used. Cognitive flexibility, Group $(-1=$ control group $/ 1=$ intervention group), and laboratory intrusions were entered as independent, moderator, and outcome variables respectively. Levels of verbal recognition memory served as control variable.

The model with laboratory intrusions as dependent variable was significant, $R^{2}=.32$, $F(4,54)=6.30, p<.001$. Analyses revealed a significant main effect of group, reflecting 
higher intrusion frequency in the control group compared to the intervention group (see Table 4).

Importantly, consistent with our hypothesis, there was a significant interactive effect of group and cognitive flexibility on the level of intrusions. This interaction accounted for an additional $14.79 \%$ of the variance beyond the variance explained by the main effect. In order to interpret the interactive effect of cognitive flexibility and group on level of intrusive symptoms, we computed bootstrapping confidence intervals (95\%) evaluating the impact of the intervention on intrusive memories for different levels of cognitive flexibility (see Table 4). As predicted, for participants with average or above-average cognitive flexibility there was a significant difference in the level of intrusive memories between the intervention and control groups. By contrast, for participants with below-average cognitive flexibility no significant group difference emerged. Figure 1 illustrates this effect for continuous levels of flexibility.

Estimated means for above-average, average and below-average cognitive flexibility revealed opposite trends in both groups (see Table 5): In the intervention group, participants with above-average flexibility experienced fewer intrusions than those with average and below-average flexibility. By contrast, in the control group, participants with below-average flexibility experienced fewer intrusions than those with average and above-average flexibility.

\section{Correlations between cognitive flexibility and intrusive memories}

Finally, we examined partial correlations between cognitive flexibility and intrusive memories controlling for experimental group. In line with our hypothesis, we found a negative correlation between cognitive flexibility and IES-R scores, $r(56)=-.29, p=.027$. However, no significant correlations were evident for ambulatory, $r(56)=-.04, p=.776$, or laboratory, $r(56)=.04, p=.759$, intrusions. 


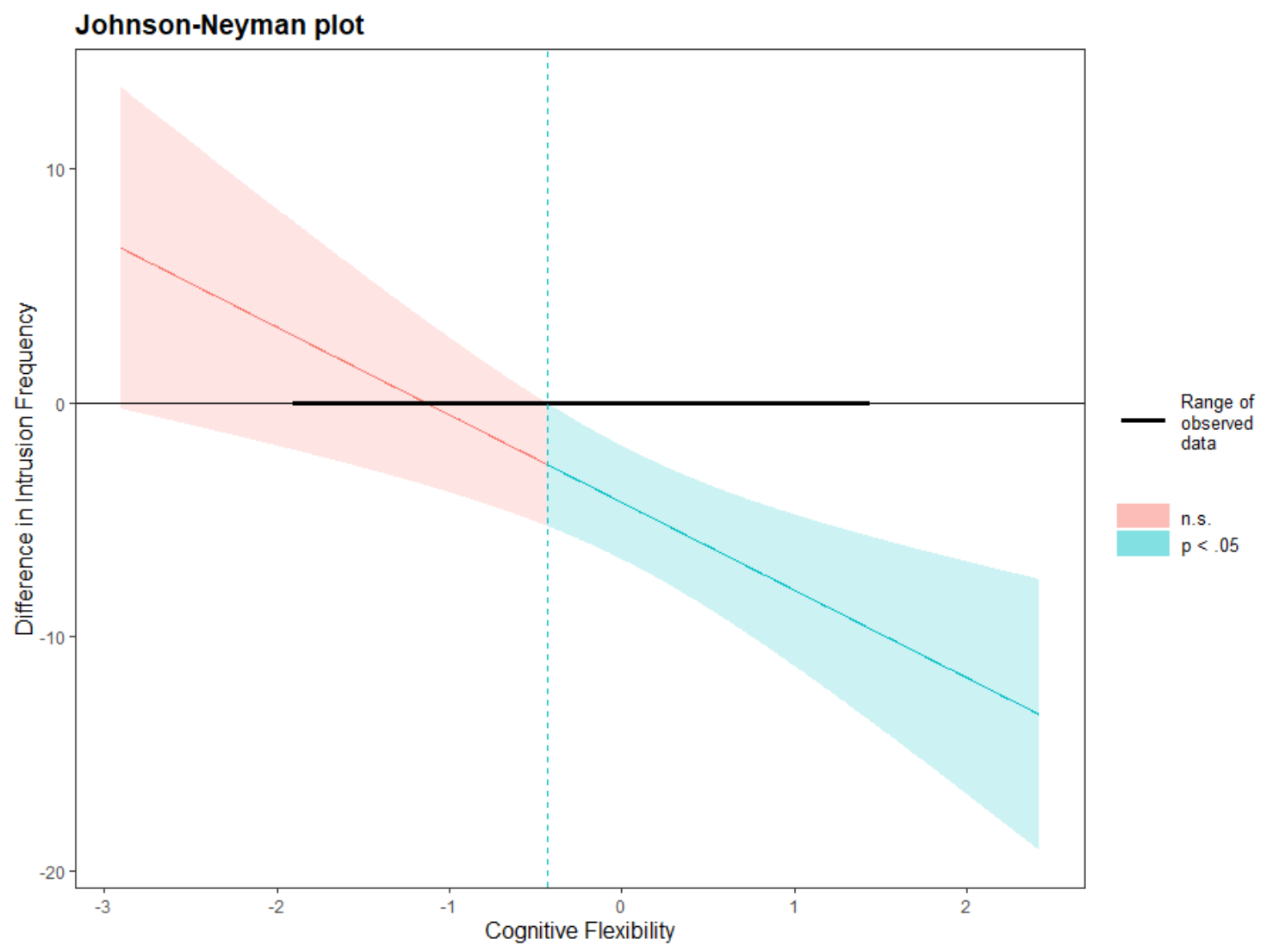

\section{Figure 1}

Johnson-Neyman plot illustrating the region of significant group differences in laboratory intrusion frequency as a function of cognitive flexibility. For the region highlighted in turquoise significant group differences emerged, whereas for the region highlighted in red no significant group differences emerged. Note: Difference in intrusion frequency reflects the absolute difference of intrusions between groups. A negative difference reflects lower intrusions in the intervention group as compared to the control group. Cognitive flexibility scores were z-standardized for ease of interpretation.

\section{Discussion}

The aim of the present study was to examine the interactive effects of cognitive flexibility and a visuospatial intervention on levels of intrusive symptoms related to posttraumatic stress following exposure to a traumatic film. In this study, there was a difference between the visuospatial intervention condition and a control condition (which involved a 
memory reactivation cue and a no task wait period) on a measure of intrusions in the laboratory, but not on intrusions as measured in daily life. As predicted, we found an interactive effect of cognitive flexibility and the visuospatial intervention on levels of laboratory intrusions. Specifically, for individuals with average and above-average cognitive flexibility, we found a significant group difference (intervention vs. control) on laboratory intrusions. No such differences were found for individuals below average in cognitive flexibility. These findings point towards a critical boundary condition of the visuospatial intervention and underline the importance of cognitive flexibility as a modulator of the efficacy of such interventions. Based on these findings and clinical research indicating that a significant proportion of PTSD patients (20\%) do not benefit from visuospatial interventions (Kessler et al., 2018), it appears warranted to further explore whether taking account of cognitive flexibility may enhance treatment outcomes of such interventions. For example, future clinical studies could screen PTSD patients for cognitive flexibility prior to treatment. If screening indicates low levels of flexibility, patients could be offered another approach or a combined intervention that trains cognitive flexibility prior to the visuospatial task.

Evaluating such a combined intervention may constitute an important step towards improving individualized treatment of PTSD.

Surprisingly, we found that in the control group highly flexible individuals seemed to have less favorable outcomes in laboratory intrusion frequency as compared to low flexible individuals. These findings may have emerged due to the specific cognitive demands during laboratory intrusion assessment. That is, after being presented with reminder cues of the traumatic film, participants were asked to let their mind wander freely, while recording the occurrence of intrusive memories. Due to the lack of cognitive demands in this period, high flexible individuals may have been at a greater risk of ruminating about the trauma film. In line with this hypothesis, a recent study by Ballesio and colleagues (2019) found a positive 
correlation between cognitive reappraisal which is highly associated with cognitive flexibility (Zaehringer et al., 2018) and rumination. Moreover, Greene (2020) found that cognitive flexibility was positively correlated with deliberate and reflective rumination. Although this form of rumination is generally not believed to have detrimental effects on mental health, it may have facilitated intrusion development under the specific cognitive demands of laboratory intrusion frequency. Less flexible individuals, on the other hand, may have been less prone to engage in deliberate rumination during the post-reminder phase, resulting in fewer intrusions. In the intervention group, such effects may have been overshadowed by the impact of the visuospatial intervention on intrusion frequency.

In addition to our main analyses, focusing on moderating effects of cognitive flexibility, we also investigated prospective associations between cognitive flexibility and intrusive memories across both groups. These analyses revealed a significant negative correlation between cognitive flexibility and IES-R scores, reflecting that higher flexibility was linked to lower IES-R scores. However, this pattern did not extend to the other measures of intrusions warranting caution in interpretation. The finding aligns with the assumption that updating flexibility - specifically for context features - might be an important factor supporting natural recovery from trauma (Kube et al., 2020). Interestingly, The IES-R intrusion subscale assesses not only intrusions but also re-experiencing symptoms in general. Moreover, it assesses the severity (i.e., intrusiveness and distress) rather than the mere frequency of these symptoms, thus best approximating clinical symptomatology of all included outcome measures.

It should be noted that in our study intervention effects were limited to laboratory intrusion frequency and did not extend to IES-R scores or to ambulatory intrusions. This finding is at odds with previous research demonstrating intervention effects for these outcomes (Holmes et al., 2009; James et al., 2015). However, it is important to note that 
ambulatory intrusion frequency $\left(\mathrm{M}_{\text {Intervention }}=0.77, \mathrm{M}_{\text {Control }}=1.27\right)$ in the current study was much lower than in previous studies (e.g., $\mathrm{M}_{\text {Intervention }}=2.69, \mathrm{M}_{\text {Control }}=5.57$ in James et al., 2016). Most notably, there was a much lower frequency of ambulatory intrusions in the control group. This pattern could reflect the presence of a floor effect in intrusion frequency that prevented us from detecting any effects driven by the intervention. A possible explanation for this floor effect could be that the film was less disturbing for Israeli participants, since many of them are exposed to various traumatic events, as a part of their daily reality and/or their military service. Furthermore, the films were all filmed in different countries than Israel (e.g., in the UK showing British road and trees) which means that triggers of intrusive memories may not have been present readily in daily life in Israel where the roads and trees look quite different. In contrast when triggers were given in the form of film stills in the IPT task, this may have led to the emergence of intrusions under more controlled laboratory condition.

Israel is a country in which civilians are often exposed to emergency situations, requiring normal coping in abnormal situations. As a result, the basic belief in the existence of a safe place might be shaken (Pat-Horenczyk, 2006). Besser and Neria (2012) examined Israelis living in differing proximity to conflict zones and found no association between psychological distress and physical proximity to a conflict zone. It is possible that since Israelis struggle to create a place-based distance from traumatic events, they mostly rely on time-based distance from such events in order to maintain their sense of wellbeing. This might explain why we could not replicate results pertaining to symptoms measured across time. In order to account for these effects, future studies in Israeli samples should use adapted stimulus material and focus on time-independent outcomes.

The present study has several limitations. First, while flexibility was measured using a well-validated performance-based paradigm, the task focused exclusively on flexible 
updating. Future studies may aim to use a set of tasks and self-report questionnaires, which measure wide and varied aspects of flexibility to test their specific role as moderator of treatment efficacy and as predictor of intrusive symptoms. Secondly, it is important to note that out study used an analog design, which limits the extent to which we can generalize our findings to the treatment of PTSD symptoms after real-world traumatic events. However, as commonly done in this field of research, we used this design in a first step to establish the potential relevance of cognitive flexibility in intrusion development. As such, our study provides an important basis for future clinically relevant research examining the moderating effect of cognitive flexibility on the efficacy of visuospatial interventions in clinical samples. Finally, since we set out to provide a first assessment of the relevance of cognitive flexibility in this field of research, our sample size was limited, and findings should be replicated in larger samples of participants.

In conclusion, the results of the current study highlight not only the beneficial effect of a visuospatial intervention on analog symptoms of post-traumatic stress, but also a boundary condition of its efficacy. That is, for individuals with low levels of cognitive flexibility no significant intervention effects were evident, suggesting the need to ameliorate flexibility deficits in this subsample prior to the administration of visuospatial interventions. These findings may pave the way for developing interventions that are tailored to individual differences between patients. Moreover, they stress the importance of flexibility to promote adaptive behavior in the face of trauma. 


\section{References}

Ågren, T., Engman, J., Frick, A., Bjorkstrand, J., Larsson, E.-M., Furmark, T., \& Fredrikson, M. (2012). Disruption of reconsolidation erases a fear memory trace in the human amygdala. Science, 337, 1550-1552. http://doi.org/10.1126/science.1223006

American Psychiatric Association (2013). Diagnostic and Statistical Manual of Mental Disorders (5th ed.). American Psychiatric Publishing.

Badawi, A., Berle, D., Rogers, K., \& Steel, Z. (2020). Do cognitive tasks reduce intrusivememory frequency after exposure to analogue trauma? An experimental replication. Clinical Psychological Science, 8(3), 569-583.

Ballesio, A., Ottaviani, C., \& Lombardo, C. (2019). Poor cognitive inhibition predicts rumination about insomnia in a clinical sample. Behavioral Sleep Medicine, 17(5), $672-681$.

Beck, A. T., Steer, R. A., Ball, R., \& Ranieri, W. F. (1996). Comparison of Beck Depression Inventories-IA and-II in psychiatric outpatients. Journal of Personality Assessment, 67(3), 588-597. https://doi.org/10.1207/s15327752jpa6703_13

Beck, A. T., Steer, R. A., \& Brown, G. K. (1996). Manual for the Beck Depression InventoryII. Psychological Corporation, 1, 82. https://doi.org/10.1007/978-94-007-07535_156

Besser, A., \& Neria, Y. (2012). When home isn't a safe haven: Insecure attachment orientations, perceived social support, and PTSD symptoms among Israeli evacuees under missile threat. Psychological Trauma: Theory, Research, Practice, and Policy, 4(1), 34-46. https://doi.org/10.1037/a0017835

Blevins, C. A., Weathers, F. W., Davis, M. T., Witte, T. K., \& Domino, J. L. (2015). The posttraumatic stress disorder checklist for DSM-5 (PCL-5): Development and initial 
psychometric evaluation. Journal of Traumatic Stress, 28(6), 489-498. https://doi.org/10.1002/jts.22059

Brennen, T., Blix, I., Nissen, A., Holmes, E. A., Skumlien, M., \& Solberg, Ø. (2021). Investigating the frequency of intrusive memories after 24 hours using a visuospatial interference intervention: a follow-up and extension. European Journal of Psychotraumatology, 12(1), 1953788.

Cañas, J. J., Fajardo, I., \& Salmeron, L. (2006). Cognitive flexibility. International Encyclopedia of Ergonomics and Human Factors, 1, 297-301. https://doi.org/10.13140/2.1.4439.6326

Delgadillo, J., Asaria, M., Ali, S., \& Gilbody, S. (2016). On poverty, politics and psychology: the socioeconomic gradient of mental healthcare utilisation and outcomes. The British Journal of Psychiatry, 209(5), 429-430. https://doi.org/10.1192/bjp.bp.115.171017

Dennis, J. P., \& Vander Wal, J. S. (2010). The cognitive flexibility inventory: Instrument development and estimates of reliability and validity. Cognitive Therapy and Research, 34(3), 241-253. https://doi.org/10.1007/s10608-009-9276-4

Garfinkel, S. N., Abelson, J. L., King, A. P., Sripada, R. K., Wang, X., Gaines, L. M., \& Liberzon, I. (2014). Impaired contextual modulation of memories in PTSD: An fMRI and psychophysiological study of extinction retention and fear renewal. Journal of Neuroscience, 34(40), 13435-13443.

Geurts, H. M., Corbett, B., \& Solomon, M. (2009). The paradox of cognitive flexibility in autism. Trends in Cognitive Sciences, 13(2), 74-82.

https://doi.org/10.1016/j.tics.2008.11.006 
Greene, T. C. (2020). Pathways to coping with extreme events: A study of the relation between cognitive flexibility and four types of rumination (Doctoral dissertation, The University of North Carolina at Charlotte).

Hagenaars, M. A., Holmes, E. A., Klaassen, F., \& Elzinga, B. (2017). Tetris and Word games lead to fewer intrusive memories when applied several days after analogue trauma. European Journal of Psychotraumatology, 8(sup1), 1386959.

Haim-Nachum, S., \& Levy-Gigi, E. (2019). A chink in the armor: the influence of training on generalization learning impairments after viewing traumatic stimuli. Cognition, 193, 104021.

Haim-Nachum, S., \& Levy-Gigi, E. (2021). The tension between cognitive and regulatory flexibility and their associations with current and lifetime PTSD symptoms. Frontiers in Psychology, 12, 1-7.

Hayes, A. F. (2017). Introduction to mediation, moderation, and conditional process analysis: A regression-based approach. Guilford publications.

Holmes, E. A., Brewin, C. R., \& Hennessy, R. G. (2004). Trauma films, information processing, and intrusive memory development. Journal of Experimental Psychology: General, 133(1), 3-22. https://doi.org/10.1037/0096-3445.133.1.3

Holmes, E. A., James, E. L., Coode-Bate, T., \& Deeprose, C. (2009). Can playing the computer game "Tetris" reduce the build-up of flashbacks for trauma? A proposal from cognitive science. PloS One, 4(1), e4153. https://doi.org/10.1371/journal.pone.0004153

Holmes, E. A., James, E. L., Kilford, E. J., \& Deeprose, C. (2010). Key steps in developing a cognitive vaccine against traumatic flashbacks: Visuospatial Tetris versus verbal Pub Quiz. PloS One, 5(11), 1-9. https://doi.org/10.1371/journal.pone.0013706 
Ionescu, T. (2012). Exploring the nature of cognitive flexibility. New Ideas in Psychology, 30(2), 190-200. https://doi.org/10.1016/j.newideapsych.2011.11.001

Iyadurai, L., Visser, R. M., Lau-Zhu, A., Porcheret, K., Horsch, A., Holmes, E. A., \& James, E. L. (2019). Intrusive memories of trauma: a target for research bridging cognitive science and its clinical application. Clinical Psychology Review, 69, 67-82.

James, E. L., Bonsall, M. B., Hoppitt, L., Tunbridge, E. M., Geddes, J. R., Milton, A. L., \& Holmes, E. A. (2015). Computer game play reduces intrusive memories of experimental trauma via reconsolidation-update mechanisms. Psychological Science, 26(8), 1201-1215. https://doi.org/10.1177/0956797615583071

James, E. L., Lau-Zhu, A., Tickle, H., Horsch, A., \& Holmes, E. A. (2016). Playing the computer game Tetris prior to viewing traumatic film material and subsequent intrusive memories: Examining proactive interference. Journal of Behavior Therapy and Experimental Psychiatry, 53, 25-33.

Katsanis, S. H., Javitt, G., \& Hudson, K. (2008). A case study of personalized medicine. Science, 320(5872), 53-54. https://doi.org/10.1126/science.1156604

Keith, J., Velezmoro, R., \& O’Brien, C. (2015). Correlates of cognitive flexibility in veterans seeking treatment for posttraumatic stress disorder. The Journal of Nervous and Mental Disease, 203(4), 287-293. https://doi.org/10.1097/NMD.0000000000000280

Kessler, H., Holmes, E. A., Blackwell, S. E., Schmidt, A. C., Schweer, J. M., Bücker, A., ... \& Kehyayan, A. (2018). Reducing intrusive memories of trauma using a visuospatial interference intervention with inpatients with posttraumatic stress disorder (PTSD). Journal of Consulting and Clinical Psychology, 86(12), 1076-1090.

Koesten, J., Schrodt, P., \& Ford, D. J. (2009). Cognitive flexibility as a mediator of family communication environments and young adults' well-being. Health Communication, 24(1), 82-94. https://doi.org/10.1080/10410230802607024 
Kube, T., Berg, M., Kleim, B., \& Herzog, P. (2020). Rethinking post-traumatic stress disorder-A predictive processing perspective. Neuroscience \& Biobehavioral Reviews, 113, 448-460.

Lau-Zhu, A., Henson, R. N., \& Holmes, E. A. (2019). Intrusive memories and voluntary memory of a trauma film: Differential effects of a cognitive interference task after encoding. Journal of Experimental Psychology: General, 148(12), 2154.

Lau-Zhu, A., Henson, R. N., \& Holmes, E. A. (2021). Selectively interfering with intrusive but not voluntary memories of a trauma film: Accounting for the role of associative memory. Clinical Psychological Science, 2167702621998315.

Leung, R. C., \& Zakzanis, K. K. (2014). Brief report: Cognitive flexibility in autism spectrum disorders: A quantitative review. Journal of Autism and Development Disorders, 44(10), 2628-2645. https://doi.org/10.1007/s10803-014-2136-4

Levy-Gigi, E., Haim-Nachum, S., Hall, J. M., Crouse, J. J., Winwood-Smith, R., Lewis, S. J., \& Moustafa, A. A. (2019). The interactive effect of valence and context on reversal learning in individuals with Parkinson's disease. Neuroscience Letters, 692, 216224.

Levy-Gigi, E., \& Richter-Levin, G. (2014). The hidden price of repeated traumatic exposure. Stress, 17(4), 343-351.

Levy-Gigi, E., Richter-Levin, G., \& Kéri, S. (2014). The hidden price of repeated traumatic exposure: different cognitive deficits in different first-responders. Frontiers in Behavioral Neuroscience, 8, 1-10.

Levy-Gigi, E., Szabo, C., Richter-Levin, G., \& Kéri, S. (2015). Reduced hippocampal Vol. is associated with overgeneralization of negative context in individuals with PTSD. Neuropsychology, 29(1), 151-161. http://dx.doi.org/10.1037/neu0000131 
Michael, T., \& Ehlers, A. (2007). Enhanced perceptual priming for neutral stimuli occurring in a traumatic context: Two experimental investigations. Behaviour Research and Therapy, 45(2), 341-358. https://doi.org/10.1016/j.brat.2006.03.012

Michael, T., Ehlers, A., Halligan, S. L., \& Clark, D. M. (2005). Unwanted memories of assault: what intrusion characteristics are associated with PTSD? Behaviour Research and Therapy, 43(5), 613-628.

Pat-Horenczyk, R. (2006). Terror in Jerusalem: Israelis coping with 'emergency routine' in daily life. Terror in the Holy Land: Inside the anguish of the Israeli-Palestinian conflict, 67-75. https://doi.org/10.1016/j.jadohealth.2005.11.021

Spielberger, C. D., Gorsuch, R. L., Lushene, R., Vagg, P. R., \& Jacobs, G. A. (1983). Manual for State-Trait Anxiety Inventory. Consulting Psychologists Press.

Topol, E. J., \& Lauer, M. S. (2003). The rudimentary phase of personalised medicine: Coronary risk scores. Lancet.

Weiss, D., \& Marmar, C. (1997). The Impact of Event Scale-Revised. W: Wilson J., Keane T.(red.). Assessing psychological trauma and PTSD: A handbook for practitioners.

Zabag, R., Deri, O., Gilboa-Schechtman, E., Richter-Levin, G., \& Levy-Gigi, E. (2020). Cognitive flexibility in PTSD individuals following nature adventure intervention: is it really that good? Stress, 23(1), 97-104.

Zaehringer, J., Falquez, R., Schubert, A. L., Nees, F., \& Barnow, S. (2018). Neural correlates of reappraisal considering working memory capacity and cognitive flexibility. Brain Imaging and Behavior, 12(6), 1529-1543.

Zlotnick, C., Warshaw, M., Shea, M. T., Allsworth, J., Pearlstein, T., \& Keller, M. B. (1999). Chronicity in posttraumatic stress disorder (PTSD) and predictors of course of comorbid PTSD in patients with anxiety disorders. Journal of Traumatic Stress: 
Official Publication of The International Society for Traumatic Stress Studies, 12(1), 89-100.

Zohar, J., Juven-Wetzler, A., Sonnino, R., Cwikel-Hamzany, S., Balaban, E., \& Cohen, H. (2011). New insights into secondary prevention in post-traumatic stress disorder.

Dialogues in Clinical Neuroscience, 13(3), 301-309. https://

doi.org/10.31887\%2FDCNS.2011.13.2\%2Fjzohar 


\section{Table 1}

Descriptive statistics and t-tests for age and baseline assessments indicating experimental groups were equivalent at baseline.

\begin{tabular}{|c|c|c|}
\hline \multirow[t]{2}{*}{ Measure } & $\begin{array}{l}\text { Visuospatial intervention } \\
\text { ( } 20 \text { female, } 10 \text { male })\end{array}$ & $\begin{array}{l}\text { Control } \\
\text { (20 female, } 10 \text { male) }\end{array}$ \\
\hline & $\mathrm{M}(\mathrm{SD})$ & $\mathrm{M}(\mathrm{SD})$ \\
\hline Age & $28.03(4.47)$ & $29.57(4.42)$ \\
\hline BDI & 5.57 (5.97) & $3.60(4.41)$ \\
\hline STAI-T & $35.60(7.30)$ & $33.50(8.23)$ \\
\hline PCL-5 & $26.87(9.76)$ & $25.13(8.25)$ \\
\hline
\end{tabular}


Table 2

Descriptive statistics for negative mood deterioration assessment for both groups.

\begin{tabular}{lcl}
\hline Measure & Visuospatial & Control \\
& intervention & \\
\cline { 2 - 3 } & $\mathrm{M}(\mathrm{SD})$ & $\mathrm{M}(\mathrm{SD})$ \\
\hline Pre-Trauma Film Mood & $1.27(0.64)$ & \\
Post-Trauma Film Mood & $3.70(2.44)$ & $4.03(3.10)$ \\
\hline
\end{tabular}




\section{Table 3}

Descriptive statistics and t-tests for traumatic symptoms and memory of the traumatic film in both experimental groups.

\begin{tabular}{|c|c|c|}
\hline \multirow[t]{2}{*}{ Measure } & Visuospatial intervention & Control \\
\hline & $\mathrm{M}(\mathrm{SD})$, range & $\mathrm{M}(\mathrm{SD})$, range \\
\hline Laboratory intrusions (IPT)* & $5.43(3.83), 0-17$ & $9.33(4.98), 2-23$ \\
\hline Ambulatory intrusions (diary) & $0.50(0.94), 0-4$ & $0.73(0.91), 0-3$ \\
\hline \multicolumn{3}{|l|}{ Day 0} \\
\hline Ambulatory intrusions (diary) & $0.77(1.83), 0-6$ & 1.27 (1.96), 0-7 \\
\hline \multicolumn{3}{|l|}{ Days 1-7 } \\
\hline IES-R intrusion subscale & $6.57(6.40), 0-21$ & 9.97 (10.34), 0-34 \\
\hline Visual Memory & $55.31(7.35), 40.63-68.75$ & $57.50(6.85), 46.88-68.75$ \\
\hline Verbal Memory* & $71.56(12.35), 43.75-93.75$ & $82.50(9.23), 46.88-93.75$ \\
\hline Film Attention & $9.03(1.30), 6-10$ & $9.07(1.17), 6-10$ \\
\hline Film Distress & $5.83(2.90), 1-10$ & 7.13 (2.69), 1-10 \\
\hline Diary Compliance & $9.20(1.32), 5-10$ & $9.10(1.12), 6-10$ \\
\hline
\end{tabular}

Note. IPT $=$ the frequency of trauma film flashbacks experienced during the initial ten minutes, Diary $=$ number of intrusions of the trauma film during a week in daily life, IES-R $=$ Impact of Events Scale intrusion subscale. Group differences were significant only for variables marked by an asterisk, $p<.01$ 


\section{Table 4}

Estimated coefficients, standard errors and $95 \%$ confidence intervals for control, independent and moderator variables in the model predicting levels of laboratory intrusions based on experimental group moderated by cognitive flexibility (context NtoP).

\begin{tabular}{lllll}
\hline Variable & $B$ & S.E. & $t$ value & $95 \%$ CI
\end{tabular}

Low High

\section{Control Variable}

$\begin{array}{llllll}\text { Verbal recognition memory } & -0.61 & 0.60 & -1.01 & -.1 .82 & 0.60\end{array}$

\section{Predictors}

\begin{tabular}{lccccc} 
Cognitive flexibility & -0.02 & 0.55 & -0.04 & -1.13 & 1.08 \\
Group & -2.12 & 0.61 & $-3.51^{*}$ & -3.34 & -0.91 \\
Group $\times$ Cognitive flexibility & -1.87 & 0.55 & $-3.42^{*}$ & -2.97 & -0.78 \\
\hline
\end{tabular}

$* p<.01$

Note . $\mathrm{CI}=$ Confidence interval, Cognitive flexibility $=$ refers to a specific measure of flexibility, Context NtoP, the ability to learn negative to positive reversions of context. 


\section{Table 5}

Conditional effect of experimental group at different levels of cognitive flexibility (context NtoP).

\begin{tabular}{llll}
\hline Cognitive flexibility & Visuospatial intervention & Control & $t$ value \\
& $\mathrm{M}$ & $\mathrm{M}$ & \\
\hline Mean - 1 SD & 7.34 & 7.85 & -0.31 \\
Mean & 5.44 & 9.69 & $-3.51^{*}$ \\
Mean + 1 SD & 3.55 & 11.54 & $-4.97^{*}$ \\
\hline$* p<.01$ & & &
\end{tabular}

Note. $\mathrm{SD}=$ Standard deviation, Cognitive flexibility $=$ refers to a specific measure of flexibility, Context NtoP, the ability to learn negative to positive reversions of context. 